
\title{
Some Properties of Interval-Valued Intuitionistic Anti Fuzzy Lie Primary Ideal
}

\author{
M. Palanivelrajan ${ }^{1 *}$, K. Gunasekaran $^{2}$ and E. Adilakshmi ${ }^{2}$ \\ 'Department of Mathematics, Government Arts College, \\ Paramakudi - 623 707, Tamil Nadu, India; palanivelrajan1975@gmail.com \\ ${ }^{2}$ Ramanujan Research Centre, PG and Research Department of Mathematics, Government Arts College \\ (Autonomous), Kumbakonam - 612 001,Tamil Nadu, India; kgunasekaran1963@gmail.com, \\ 1985adilakshmi@gmail.com
}

\begin{abstract}
In this paper, the theory of fuzzy semiprimary ideal [16] is extended by introducing intuitionistic anti fuzzy primary ideals as well as intuitionistic anti fuzzy semiprimary ideals in rings. Similarly, Interval-Valued Intuitionistic Anti Fuzzy Lie Primary Ideals (IVIAFLPI) is defined. Various properties of IVIAFLPI are discussed. Finally, Interval-Valued Intuitionistic Fuzzy Lie Semiprimary Ideals (IVIAFLSPI) is established.
\end{abstract}

Keywords: Intuitionistic Fuzzy Set, Intuitionistic Anti Fuzzy Ideal, Intuitionistic Anti Fuzzy Primary Ideal, Intuitionistic Anti Fuzzy Semi-Primary Ideal, Interval-Valued Intuitionistic Anti Fuzzy Lie Primary Ideals

\section{Introduction}

After the introduction of Fuzzy Sets (FSs) by A. Zadeh [12], the fuzzy concept has been used to extend almost all areas of mathematics. By using FSs people have recognized the theory to study uncertainty. Fuzzy mathematics have become a vital area of research in different applications such as engineering, medical science, social science, artificial intelligence, signal processing, pattern recognition, computer networks, automata theory and so on. The notion of IFS and its operations were introduced by Atanassov [1], as a generalization of the concept of FS. Atanassov [2] discussed the operators over IntervalValued Intuitionistic Fuzzy Sets (IVIFSs). Palanivelrajan and Nandakumar [9] introduced the definition and some properties of intuitionistic fuzzy primary and semiprimary ideals [16].

The notion of Lie groups [13] was first introduced by sophus Lie in nineteenth century through his studies in geometry and integration methods for solving differential equations. Lie algebras [6] were also exposed by him when he attempted to categorize certain smooth subgroups of a general linear group. In applied mathematics Lie theory [13] remains a commanding tool for studying differential equations, special functions and perturbation theory. It is noted that Lie theory has applications in physics also. M. Akram. W. A. Dudek [7] discussed Intervalvalued intuitionistic fuzzy Lie ideals of Lie algebras. P. K. Sharma [10], discussed intuitionistic Anti fuzzy ideal and Quotient ring.

In this paper, interval-valued intuitionistic anti fuzzy lie primary ideals and anti fuzzy Lie ideals, interval-valued intuitionistic anti fuzzy Lie ideals of Lie algebras are discussed.

\section{Preliminaries}

In this section, some basic definitions which are necessary for this paper are presented.

Definition 2.1 [11]

A fuzzy subset $\mu$ of a ring $R$ is called fuzzy ideal if for all $x, y \in R$ the subsequent conditions are satisfied

${ }^{*}$ Author for correspondence 
(i) $\quad \mu(x-y) \geq \min (\mu(x), \mu(y))$

(ii) $\quad \mu(x y) \geq \max (\mu(x), \mu(y))$

\section{Example 2.1}

$$
\mu= \begin{cases}1, & \text { if } x=0 \\ 0.8, & \text { if } x \in\langle 4\rangle \sim\langle 0\rangle \\ 0.6, & \text { if } x \in z \sim\langle 4\rangle\end{cases}
$$

Take $x=4, y=3, x-y=1$

$$
\begin{aligned}
& (i) \mu_{A}(x-y) \geq \min \left(\mu_{A}(x), \mu_{A}(y)\right) \\
& \mu_{A}(1) \geq \min \left(\mu_{A}(4), \mu_{A}(3)\right) \\
& \mu_{A}(1) \geq \min (0.8,0.6) \\
& 0.6 \geq 0.6
\end{aligned}
$$

$$
\begin{aligned}
& (i i) \mu_{A}(x y) \geq \max \left(\mu_{A}(x), \mu_{A}(y)\right) \\
& \mu_{A}(12) \geq \max \left(\mu_{A}(4), \mu_{A}(3)\right) \\
& \mu_{A}(12) \geq \max (0.8,0.6) \\
& 0.8 \geq 0.8
\end{aligned}
$$

Definition 2.2 [14]

A fuzzy subset $\mu$ of a ring $R$ is called anti fuzzy ideal if for all $x, y \in R$ the subsequent conditions are satisfied

(i) $\quad \mu(x-y) \leq \max (\mu(x), \mu(y))$

(ii) $\quad \mu(x y) \leq \min (\mu(x), \mu(y))$.

\section{Definition 2.3 [14]}

A fuzzy subset $\mu$ of a ring $R$ is called intuitionistic anti fuzzy ideal if for all $x, y \in R$, the subsequent conditions are satisfied,

$$
\mu_{A}(x-y) \leq \max \left(\mu_{A}(x), \mu_{A}(y)\right)
$$

$$
\begin{aligned}
& \mu_{A}(x y) \leq \min \left(\mu_{A}(x), \mu_{A}(y)\right) \\
& \gamma_{A}(x-y) \geq \min \left(\gamma_{A}(x), \gamma_{A}(y)\right) \\
& \gamma_{A}(x y) \geq \max \left(\gamma_{A}(x), \gamma_{A}(y)\right)
\end{aligned}
$$

\section{Example 2.2}

Let $\mathrm{R}=\mathrm{Z}$, the ring of integers under ordinary addition and multiplication of integers.

Define the two IFS's A and B by

$$
\mu_{A}(x)= \begin{cases}0.5, & \text { if } x \text { is a multiple of } 3 \\ 1, & \text { otherwise }\end{cases}
$$

$$
\mu_{B}(x)=\left\{\begin{array}{l}
0.8, \text { if } x \text { is a multiple of } 3 \\
0.83, \text { otherwise }
\end{array}\right.
$$

and

$$
\gamma_{A}(x)= \begin{cases}0.3, & \text { if } x \text { is a multiple of } 3 \\ 0, & \text { otherwise }\end{cases}
$$

$$
\gamma_{B}(x)=\left\{\begin{array}{l}
0.15, \text { if } x \text { is a multiple of } 3 \\
0.05, \text { otherwise }
\end{array}\right.
$$

It can be easily verified that $A$ and $B$ are IAFI of $Z$.

\section{Definition 2.4}

An intuitionistic anti fuzzy ideal $R$ of a ring $R$ is called Intuitionistic anti fuzzy primary ideal (IAFPI) if for all $a, b \in R$ either $\mu_{A}(a b)=\mu_{A}(a)$ and $\gamma_{A}(a b)=\gamma_{A}(a)$, or $\mu_{A}(a b) \geq \mu_{A}\left(b^{m}\right)$ and $\gamma_{A}(a b)$ $\leq \gamma_{A}\left(b^{m}\right)$, for some $m \in Z^{+}$.

\section{Example 2.3}

$$
\mu_{A}(x)= \begin{cases}1, & \text { if } x=0 \\ 0.8, & \text { if } x \in\langle 4\rangle \sim\langle 0\rangle \\ 0.6, & \text { if } x \in z \sim\langle 4\rangle\end{cases}
$$

and

$$
\gamma_{A}(x)= \begin{cases}0, & \text { if } x=0 \\ 0.1, & \text { if } x \in\langle 4\rangle \sim\langle 0\rangle \\ 0.3, & \text { if } x \in z \sim\langle 4\rangle\end{cases}
$$

Consider $0,4 \in z$

$$
\begin{aligned}
& \mu_{A}(0.4)=\mu_{A}(0) \Rightarrow 1=1 \\
& \gamma_{A}(0.4)=\gamma_{A}(0) \Rightarrow 0=0 \\
& \mu_{A}(3.4)=\mu_{A}(3) \Rightarrow 0.6=0.6 \\
& \gamma_{A}(3.4)=\gamma_{A}(3) \Rightarrow 0.3=0.3
\end{aligned}
$$

Now $\mu_{A}(0.4) \geq \mu_{A}\left(4^{m}\right) \Rightarrow 1 \geq 0.8$

$$
\begin{aligned}
& \gamma_{A}(0.4) \leq \gamma_{A}\left(4^{m}\right) \Rightarrow 0 \leq 0.1 \\
& \mu_{A}(4.3) \geq \mu_{A}\left(3^{m}\right) \Rightarrow 0.6 \geq 0.6 \\
& \gamma_{A}(4.3) \leq \gamma_{A}\left(3^{m}\right) \Rightarrow 0.3 \leq 0.3
\end{aligned}
$$




\section{Definition 2.5}

An intuitionistic anti-fuzzy ideal $A$ of a ring $R$ is called Intuitionistic Anti Fuzzy Semiprimary Ideal (IAFSPI) if for all $a, b \in R$ either $\mu_{A}(a b) \geq \mu_{A}\left(a^{n}\right)$ and $\gamma_{A}(a b) \leq \gamma_{A}\left(a^{n}\right)$, for some $n \in Z^{+}$or else $\mu_{A}(a b)$ $\geq \mu_{A}\left(b^{m}\right)$ and $\gamma_{A}(a b) \leq \gamma_{A}\left(b^{m}\right)$ for some $m \in Z^{+}$.

\section{Definition 2.6 [2]}

An interval-valued fuzzy set $A$ is specified by a function $M_{A}: E \rightarrow D[0,1]$, where $D[0,1]$ is the set of all intervals within $[0,1]$, for all $x \in E, M_{A}(x)$ is an interval $[a, b]$, where $0 \leq a \leq b \leq 1$.

\section{Definition 2.7 [2]}

An interval-valued intuitionistic fuzzy set $A$ over $E$ is defined as an object of the form $A=\left\{x, M_{A}(x)\right.$, $\left.N_{A}(x) \mid x \in E\right\}$, where $M_{A}(x) \subseteq[0,1]$ and $N_{A}(x)$ $\subseteq[0,1]$ are interval and for all $x \in E, \operatorname{rmax} M_{A}$ $\operatorname{rmax}_{A}(x)+\operatorname{rmax}_{A}(x) \leq 1$.

\section{Definition 2.8}

Let $\mathrm{E}_{1}$ and $\mathrm{E}_{2}$ be two universes and let $A=\left\{x, \mu_{A}(x), \gamma_{A}(x) / x \in E_{1}\right\}, B=\left\{y, \mu_{B}(y), \gamma_{B}\right.$ $\left.(x) / y \in E_{2}\right\}$ be two interval-valued intuitionistic fuzzy subsets of $\mathrm{E}_{1}$ and $\mathrm{E}_{2}$ respectively, then

$$
\begin{gathered}
A \times B=\left\{(x, y), \min \left(\operatorname{rmin} M_{A}(x), \operatorname{rmin}\right.\right. \\
\left.M_{B}(y)\right), \max \left(\operatorname{rmin}_{A}(x), \operatorname{rmin}_{B}(y)\right) \\
\left./ x \in E_{1} \text { and } y \in E_{2}\right\}
\end{gathered}
$$

\section{Definition 2.9}

Let $A$ be an interval-valued intuitionistic fuzzy sets. A fuzzy ideal $A$ of a ring $R$ is said to be interval-valued intuitionistic anti fuzzy primary ideal (IVIAFPI) of $R$ if for all $a, b \in R$ then either $\mu_{A}(a b)=\operatorname{rmax} M_{A}(a b)=\operatorname{rmax} M_{A}(a)$

$$
=\mu_{A}(a) \quad \text { and } \quad \gamma_{A}(a b)=\operatorname{rmax} N_{A}(a b)
$$

$$
\begin{aligned}
& =\operatorname{rmax} N_{A}(a)=\gamma_{A}(a) \text { or } \mu_{A}(a b)=\operatorname{rmax} \mathrm{M}_{A}(a b) \\
& \geq \operatorname{rmax} \mathrm{M}_{A}\left(b^{n}\right)=\mu_{A}\left(b^{n}\right) \text { and } \gamma_{A}(a b)=\operatorname{rmax} \\
& V_{A}(a b) \leq \operatorname{rmax} N\left(b^{n}\right)=\gamma_{A}\left(b^{n}\right), \text { for some } n \in Z^{+} .
\end{aligned}
$$

\section{Definition 2.10}

Let $A$ be an interval-valued intuitionistic fuzzy set $\mathrm{A}$ fuzzy ideal $A$ of a ring $R$ is said to be Interval-Valued Intuitionistic Anti Fuzzy Semiprimary Ideal (IVIAFSPI) of $R$ if for all $a, b \in R$ then either

$$
\begin{gathered}
\mu_{A}(a b)=\operatorname{rmax}_{A}(a b) \geq \operatorname{rmax}_{A}\left(a^{n}\right) \\
=\mu_{A}\left(a^{n}\right) \text { and } \gamma_{A}(a b)=\operatorname{rmax}_{A}(a b) \\
\leq \operatorname{rmax}_{A}\left(a^{n}\right)=\gamma_{A}\left(a^{n}\right), \text { for some } \\
n \in Z^{+} \operatorname{or} \mu_{A}(a b)=\operatorname{rmax} M_{A}(a b) \geq \operatorname{rmax} \\
\mu_{A}\left(b^{m}\right)=\mu_{A}\left(b^{m}\right) \text { and } \gamma_{A}(a b)=\operatorname{rmax} \\
N_{A}(a b) \leq \operatorname{rmax}_{A}\left(b^{m}\right)=\gamma_{A}\left(b^{m}\right), \text { for some } \\
m \in Z^{+} .
\end{gathered}
$$

\section{Definition 2.11 [18]}

A fuzzy set $\mu: L \rightarrow[0,1]$ is called a fuzzy Lie subalgebra of $\mathrm{L}$ if

(i) $\mu(x+y) \geq \min (\mu(x), \mu(y))$

(ii) $\mu(a x) \geq \mu(x)$

(iii) $\mu([x y]) \geq \min (\mu(x), \mu(y))$ holds for all $x, y \in L$ and $a \in F$.

Definition 2.12 [7]

A fuzzy set $\mu: L \rightarrow[0,1]$ is called anti fuzzy Lie ideal of $L$ if

(i) $\mu(x+y) \leq \max (\mu(x), \mu(y))$

(ii) $\mu(a x) \leq \mu(x)$

(iii) $\mu([x y]) \leq \mu(x)$ holds for all $x, y \in L$ and $a \in F$. 


\section{Interval-valued Intuitionistic Anti Fuzzy Lie Primary Ideal}

\section{Definition 3.1}

An IVIFS $A=\left(\mu_{A}, \gamma_{A}\right)$ in $\mathrm{L}$ is called an Interval-Valued Intuitionistic Anti Fuzzy Lie Ideal (IVIAFLI) of L, if the following conditions are satisfied.

(i) $\quad \bar{\mu}_{A}(x+y) \leq \max \left(\bar{\mu}_{A}(x), \bar{\mu}_{A}(y)\right)$ and $\bar{\gamma}_{A}(x+y) \geq \min \left(\bar{\gamma}_{A}(x), \bar{\gamma}_{A}(y)\right)$

(ii) $\bar{\mu}_{A}(a x) \leq \bar{\mu}_{A}(x)$ and $\bar{\gamma}_{A}(a x) \geq \bar{\gamma}_{A}(x)$

(iii) $\bar{\mu}_{A}([x+y]) \leq \bar{\mu}_{A}(x)$ and

$\bar{\gamma}_{A}([x+y]) \geq \bar{\gamma}_{A}(x)$ for all $x, y \in L$ and $a \in F$

\section{Definition 3.2}

Let $A$ be an interval-valued intuitionistic anti fuzzy Lie ideal of a Lie algebra L then A is said to be an Interval-Valued Intuitionistic Anti Fuzzy Lie Primary Ideal (IVIAFLPI) of $\mathrm{L}$ if for all $x, y \in L$ then either $\bar{\mu}_{A}(x y)=\bar{\mu}_{A}(x)$ and $\bar{\gamma}_{A}(x y)=\bar{\gamma}_{A}(x)$ or $\bar{\mu}_{A}(x y) \geq \bar{\mu}_{A}\left(x^{n}\right)$ and $\bar{\gamma}_{A}(x y) \leq \bar{\gamma}_{A}\left(x^{n}\right)$ for some $n \in Z^{+}$.

\section{Definition 3.3}

Let $\mathrm{A}$ be an IVIAFLPI of a Lie algebra $\mathrm{L}$ then $\mathrm{A}$ is said to Interval-Valued Intuitionistic Anti Fuzzy Lie Semiprimary Ideal (IVIAFLSPI) of L if for all $x, y \in L$ and for some $n \in Z^{+}$. Either $\bar{\mu}_{A}(x y) \geq \bar{\mu}_{A}\left(x^{n}\right)$ and $\bar{\gamma}_{A}(x y) \leq \bar{\gamma}_{A}\left(x^{n}\right)$ or else $\bar{\mu}_{A}(x y) \geq \bar{\mu}_{A}\left(y^{m}\right)$ and $\bar{\gamma}_{A}(x y) \leq \bar{\gamma}_{A}\left(y^{m}\right)$ for some $m \in Z^{+}$.

\section{Theorem 3.1 [13] [17]}

If $A=\left(\bar{\mu}_{A}, \bar{\gamma}_{A}\right)$ is an IVIAFLPI of a lie algebra $\mathrm{L}$, then the anti level subset $U\left(\bar{\mu}_{A}, \bar{a}\right)=\left\{\alpha \in L / \bar{\mu}_{A}(x) \leq \bar{a}\right\}$ and $L\left(\bar{\gamma}_{A}, \bar{a}\right)=\left\{x \in L / \bar{\gamma}_{A}(x) \geq \bar{a}\right\}$ are lie primary ideals of $\mathrm{L}$ for every $\bar{a} \in I_{m}\left(\bar{\mu}_{A}\right) \cap I_{m}\left(\bar{\gamma}_{A}\right) \subseteq D[0,1]$, where $I_{m}\left(\bar{\mu}_{A}\right)$ and $I_{m}\left(\bar{\gamma}_{A}\right)$ are sets of values of $\bar{\mu}_{A}$ and $\bar{\gamma}_{A}$ respectively.

\section{Proof:}

Let $\quad \bar{\alpha} \in I_{m}\left(\bar{\mu}_{A}\right) \cap I_{m}\left(\bar{\gamma}_{A}\right) \subseteq D[0,1]$, and let $x, y \in \bigcup\left(\bar{\mu}_{A}, \bar{a}\right)$ and $a \in F$, then $\bar{\mu}_{A}(x) \leq I$, where $I=[0,1]$ and $\bar{\mu}_{A}(x) \leq \bar{\alpha}$ it follows that $\bar{\mu}_{A}(x y)=\bar{\mu}_{A}(x) \leq \bar{a}$, so that $x, y \in U\left(\bar{\mu}_{A}, \bar{a}\right)$, consequently $U\left(\bar{\mu}_{A}, \bar{a}\right)$ IVIAFLPI of L. Let $x, y \in L\left(\bar{\gamma}_{A}, \bar{a}\right)$ and $a \in F$, then $\bar{\gamma}_{A}(x) \leq I$ where $I=[0,1]$ and $\bar{\gamma}_{A}(x) \geq \bar{\alpha}$, it follows that $\bar{\gamma}_{A}(x y)=\bar{\gamma}_{A}(x)$, so that $x, y \in L\left(\bar{\gamma}_{A}, \bar{a}\right)$ consequently $L\left(\bar{\gamma}_{A}, \bar{a}\right)$ is IVIAFLPI of $\mathrm{L}$.

\section{Theorem 3.2 [13] [14] [16] [17]}

If $A=\left(\bar{\mu}_{A}, \bar{\gamma}_{A}\right)$ and $B=\left(\bar{\mu}_{B}, \bar{\gamma}_{B}\right)$ be two IVIAFLPI of a Lie algebra $L$, then $A \times B$ is an IVIAFLPI of $L \times L$.

\section{Proof:}

We know that $A \times B=\left\{\bar{\mu}_{A} \times \bar{\mu}_{A}, \bar{\gamma}_{B} \times \bar{\gamma}_{B}\right\}$

where $\left(\bar{\mu}_{A} \times \bar{\mu}_{B}\right)(x, y)=\max \left(\bar{\mu}_{A}(x), \bar{\mu}_{B}(y)\right)$

and $\left(\bar{\gamma}_{A} \times \bar{\gamma}_{B}\right)(x, y)=\min \left(\bar{\gamma}_{B}(x), \bar{\gamma}_{B}(y)\right)$

Let $x=\left(x_{1}, x_{2}\right)$ and $y=\left(y_{1}, y_{2}\right) \in L \times L$

Now

$$
\begin{aligned}
\left(\bar{\mu}_{A} \times \bar{\mu}_{B}\right)(x y) & =\bar{\mu}_{A} \times \bar{\mu}_{B}\left(\left(x_{1}, x_{2}\right)\left(y_{1}, y_{2}\right)\right)=\bar{\mu}_{A} \times \bar{\mu}_{B}\left(x_{1} y_{1}, x_{2} y_{2}\right) \\
& =\max \left(\bar{\mu}_{A}\left(x_{1} y_{1}\right), \bar{\mu}_{B}\left(x_{2} y_{2}\right)\right) \\
& =\max \left(\bar{\mu}_{A}\left(x_{1}\right), \bar{\mu}_{B}\left(x_{2}\right)\right) \\
& =\left(\bar{\mu}_{A} \times \bar{\mu}_{B}\right)\left(x_{1}, x_{2}\right) \\
& =\left(\bar{\mu}_{A} \times \bar{\mu}_{B}\right)(x)
\end{aligned}
$$

Therefore $\left(\bar{\mu}_{A} \times \bar{\mu}_{B}\right)(x y)=\left(\bar{\mu}_{A} \times \bar{\mu}_{B}\right)(x)$

Now

$$
\begin{aligned}
\left(\bar{\gamma}_{A} \times \bar{\gamma}_{B}\right)(x y) & =\left(\bar{\gamma}_{A} \times \bar{\gamma}_{B}\right)\left(\left(x_{1}, x_{2}\right)\left(y_{1}, y_{2}\right)\right) \\
& =\left(\bar{\gamma}_{A} \times \bar{\gamma}_{B}\right)\left(x_{1} y_{1}, x_{2} y_{2}\right) \\
& =\min \left(\bar{\gamma}_{A}\left(x_{1} y_{1}\right), \bar{\gamma}_{B}\left(x_{2} y_{2}\right)\right) \\
& =\min \left(\bar{\gamma}_{A}\left(x_{1}\right), \bar{\gamma}_{B}\left(x_{2}\right)\right) \\
& =\left(\bar{\gamma}_{A} \times \bar{\gamma}_{B}\right)\left(x_{1} x_{2}\right) \\
& =\left(\bar{\gamma}_{A} \times \bar{\gamma}_{B}\right)(x) .
\end{aligned}
$$

Therefore, $\left(\bar{\gamma}_{A} \times \bar{\gamma}_{B}\right)(x y)=\left(\bar{\gamma}_{A} \times \bar{\gamma}_{B}\right)(x)$ and hence $A \times B$ is an IVIAFLPI of $\mathrm{L}$. 
Theorem 3.3 [13] [14] [16] [18]

If $A=\left(\bar{\mu}_{A}, \bar{\gamma}_{A}\right)$ and $B=\left(\bar{\mu}_{B}, \bar{\gamma}_{B}\right)$ are IVIAFLPI on $\mathrm{L}$ then $[A, B]$ is also an IVIAFLPI of $\mathrm{L}$.

\section{Proof:}

Let $\mathrm{A}$ be an IVIAFLPI of a Lie algebra $\mathrm{L}$ then $\bar{\mu}_{A}(x y)=\bar{\mu}_{A}(x)$ and $\bar{\gamma}_{A}(x y)=, \bar{\gamma}_{A}(x)$, for some every $\mathrm{x}, \mathrm{y} \in \mathrm{L}$.

Consider $x, y \in L$.

Now

$$
\begin{gathered}
<\bar{\mu}_{A}, \bar{\mu}_{B}>>(x y)=r \min \left(\max \left(\bar{\mu}_{A}(x y), \bar{\mu}_{B}(x y)\right)\right. \\
\left./ x y, x, y \in L_{1},[x y, x y]=x y\right) \\
=r \min \left(\max \left(\bar{\mu}_{A}(x), \bar{\mu}_{B}(x)\right)\right) \\
=<<\bar{\mu}_{A}, \bar{\mu}_{B}>>(x)
\end{gathered}
$$

Therefore,

$$
<<\bar{\mu}_{A}, \bar{\mu}_{B}>>(x y)=.<<\bar{\mu}_{A}, \bar{\mu}_{B}>>(x)
$$

Now

$$
\begin{gathered}
\ll<\bar{\gamma}_{A}, \bar{\gamma}_{B} \gg(x y)=r \max \left(\min \left(\bar{\gamma}_{A}(x y), \bar{\gamma}_{B}(x y)\right)\right. \\
\left.\mid x y, x, y \in L_{1},[x y, x y]=x y\right) \\
=r \max \left(\min \left(\bar{\gamma}_{A}(x), \bar{\gamma}_{B}(x)\right)\right) \\
=<<\bar{\gamma}_{A}, \bar{\gamma}_{B}>>(x)
\end{gathered}
$$

Therefore,

$$
<<\bar{\gamma}_{A}, \bar{\gamma}_{B}>>(x y)=<<\bar{\gamma}_{A}, \bar{\gamma}_{B}>>(x)
$$

Therefore, $[A, B]$ is an IVIAFLPI of $L$.

\section{Theorem 3.4 [13]}

If $\mathrm{A}_{1}, \mathrm{~A}_{2}, \mathrm{~B}_{1}, \mathrm{~B}_{2}$ be IVIAFLPI in $\mathrm{L}$ such that $A_{1} \supseteq A_{2}$ and $B_{1} \supseteq B_{2}$ then $\left[A_{1}, B_{1}\right] \supseteq\left[A_{2}, B_{2}\right]$.

\section{Proof:}

Consider $x, y \in L$

Now,

$$
\begin{gathered}
\ll<\bar{\mu}_{A 1}, \bar{\mu}_{B 1} \gg(x y)=r \min \left(\max \left(\bar{\mu}_{A 1}(x y), \bar{\mu}_{B 1}(x y)\right)\right. \\
\left./ x y, x, y \in L_{1},[x y, x y]=x y\right) \\
=r \min \left(\max \left(\bar{\mu}_{A 1}(x y), \bar{\mu}_{B 1}(x y)\right)\right.
\end{gathered}
$$

$$
\begin{gathered}
\leq r \min \left(\max \left(\bar{\mu}_{A 2}(x y), \bar{\mu}_{B 2}(x y)\right)\right. \\
\left.\quad x y, x, y \in L_{1},[x y, x y]=x y\right) \\
=r \min \left(\max \left(\bar{\mu}_{A 2}(x), \bar{\mu}_{B 2}(x)\right)\right. \\
=<<\bar{\mu}_{A 2}, \bar{\mu}_{B 2}>>(x)
\end{gathered}
$$

Therefore,

$$
<<\bar{\mu}_{A 1}, \bar{\mu}_{B 1}>>(x y)=<<\bar{\mu}_{A 2}, \bar{\mu}_{B 2}>>(x)
$$

Now

$$
<<\bar{\gamma}_{A 1}, \bar{\gamma}_{B 1}>>(x y)=
$$

$$
\begin{gathered}
r \max \left(\min \left(\bar{\gamma}_{A 1}(x y), \bar{\gamma}_{B 1}(x y)\right) / x y, x, y \in L_{1},[x y, x y]=x y\right) \\
\geq r \max \left(\min \left(\bar{\gamma}_{A 2}(x y), \bar{\gamma}_{B 2}(x y)\right)\right. \\
\left./ x y, x, y \in L_{1},[x y, x y]=x y\right) \\
=r \max \left(\min \left(\bar{\gamma}_{A 2}(x y), \bar{\gamma}_{B 2}(x y)\right)\right. \\
=<<\bar{\gamma}_{A 2}, \bar{\gamma}_{B 2}>(x)
\end{gathered}
$$

Therefore,

$$
\begin{aligned}
& <<\bar{\gamma}_{A 1}, \bar{\gamma}_{B 1}>>(x y)=.<<\bar{\gamma}_{A 2}, \bar{\gamma}_{B 2}>>(x) \\
& \text { Hence, }\left[A_{1}, B_{1}\right] \supseteq\left[A_{2}, B_{2}\right] .
\end{aligned}
$$

\section{References}

1. Atanassov K. T., "Intuitionistic fuzzy sets", Fuzzy Sets and Systems, Vol. 20(1), p. 87-96, 1986.

2. Atanassov K., "Operators over interval-valued intuitionistic fuzzy sets", Fuzzy Sets and Systems, Vol. 64(2), p. 159-174, 1994.

3. Chakrabarty K., Biswas R., Nanda S., "A note on union and intersection of intuitionistic fuzzy sets", Notes on Intuitionistic Fuzzy Sets, Vol. 3(4), 1997.

4. Deschrijver G., "Arithmetic operators in interval-valued fuzzy theory", Information Sciences, Vol. 177, p. 2906-2924, 2007.

5. Humphreys J. E., Introduction to lie algebras and representation theory, springer, New York.

6. Qin K., Qiao Q., Chen C., "Some properties of fuzzy Lie algebras". The Journal of Fuzzy Mathematics, Vol. 9(4), p. 985-989, 2001.

7. Akram M., Dudek W. A., "Interval-valued intuitionistic fuzzy Lie ideals of Lie algebras", World Applied Sciences Journal, Vol. 7, p. 812-819, 2009. 
8. Bhowmik M., Pal M., "Generalized interval-valued intuitionistic fuzzy sets", International Journal Fuzzy Mathematics, Vol. 18(2), p. 357-371, 2010.

9. Palanivelrajan M., Nandakumar S., "Some properties of intuitionistic fuzzy primary and semiprimary ideals", Notes on Intuitionistic Fuzzy Sets, Vol. 18(3), p. 68-74, 2012.

10. Sharma P. K., "On intuitionistic Anti-fuzzy ideal and Quotient ring, Global Research publications”, Vol. 4(2), p. 109-119, 2012.
11. Rajesh K., "Fuzzy semiprimary ideals of rings", Fuzzy Sets and Systems, Vol. 42, p. 263-272, 1991.

12. Zadeh L. A., "Fuzzy sets", Information and Control, Vol. 8, p. 338-353, 1965.

13. Available from: www.idosi.org

14. Available from: www.ijmttjournal.org

15. Available from: www.scialert.net

16. Available from: www.ifigenia.org

17. Available from: www.m-hikari.com

18. Available from: www.quasigroups.eu 